\title{
Comparison of UV Concentration Geometries as an Element Capable for the Increment of the Photocatalytic Efficiency of Advanced Oxidation Processes, Inside the Annular Batch Systems
}

\author{
Tonahtiuh Rendon ${ }^{1 *}$, Fernando Hernandez ${ }^{2}$ and Juan Castillo ${ }^{3}$
}

${ }^{1}$ Laboratory of Environmental Chemistry, ICUAP, Pubela, Mexico

${ }^{2}$ Environmental Chemistry Academic Body, Benemérita Autonomous University of Puebla, Mexico

${ }^{3}$ Faculty of Physical and Mathematical Sciences, Benemérita Autonomous University of Puebla, México

\begin{abstract}
The present research examines the efficiency achieved by the implementation of two collectors of ultraviolet radiation in an annular batch type photochemical reactor. The first geometry corresponds to a concentric cylinder with the source of radiation, which consists in a media mercury lamp with 1000 watts of power. The second dispositive is constitute by the implementation of two compound parabolic collectors, opposed in their aperture areas, with a concentration factor equal to one and an acceptance angle of $90^{\circ}$. The obtained results shows the capacity of both geometries to increase the efficiency of the system during the Blue Erionyl A-R acid dye degradation, dissolved in aqueous media, by the application of Advanced Oxidation Processes, in an homogeneous phase of ultraviolet radiation and $\mathrm{H}_{2} \mathrm{O}_{2}$ catalyst application.

By the use of $0.05 \mathrm{~mL}$ per liter of hydrogen peroxide at $50 \%$ as a $\bullet \mathrm{OH}$ radicals source, and a colorant concentration of $50 \mathrm{ppm}$, the collectors allows the 98.4 and $100 \%$ of the dye photochemical degradation into a experimental time of 5 minutes, So the experiments registering a system efficiency increasing around of 3.4 and $5.5 \%$ respectively. Unlike the original system, and of the cylindrical collector application, the opposite compound parabolic concentrators showed a better capacity to degrade the intermediate products, that are been produced during the mineralization process of the textile compound and absorbs light radiation at wavelengths included between 230 and $480 \mathrm{~nm}$. This promotes a water treatment process time decrement and an electrical energy savings.
\end{abstract}

Keywords: Advanced oxidation processes; Compound parabolic concentrators; Photochemical batch system

\section{Introduction}

The Advanced Oxidation Processes (AOP's) have been widely used for the degradation or pre-treatment of diverse chemical pollutants that could hardly be treated by other physical or biological methods [1].

Regardless of whether they are performed in liquid or gaseous phase, their operation are based on the generation of potent oxidant species that are highly reactive and unselective (hydroxyl radicals: - $\mathrm{OH}$ ) from the photolysis and reaction of some kind of catalyst (such as $\mathrm{H}_{2} \mathrm{O}_{2}, \mathrm{O}_{3}, \mathrm{TiO}_{2}, \mathrm{Fe}^{2+}$, etc.) with the medium in which they are dissolved.

Once produced, the $\bullet \mathrm{OH}$ radicals reacts in a chain form with the different pollutants and changing its molecular structure, promotes their mineralization process with the only generation of water, carbon dioxide or mineral salts residues, in the best of the cases.

Usually developed under ultraviolet (UV) radiation, the AOP's have reported the chemical products removal like anilines [2] textile dyes [3] sulphurous species [4] bio-recalcitrant pesticides [5] herbicides, fungicides [6], phenols [7] pharmaceutical formulations [8] among other compounds [9] in a successfully form. Therefore, the AOP's can be considered as a destructive sink of these undesirable compounds in the environment, and can be scaled or automated for both the increment of the water volume treatment and of the efficiency of the operation.

The artificial radiation UV sources employed in the AOP's are commonly formed by the low (LP-UV) and medium (MP-UV) steam pressure mercury lamps, or by the use of arc Xenon pulsed lamps [10]. One of its possible physical configurations is showed in Figure 1. It displays a batch type system, whose MP-UV source is located on the photo-reactor axe (annular configuration). Concentric and external to it, there are two quartz tubes with a transmission of $99 \%$ in the UV region. The smallest of them (in diameter) allows the circulation of the coolant liquid (consisting of demineralized water), for the lamp temperature control. The second contains both the liquid to be treated and a magnetic stirrer (at the bottom of the container) to facilitate the turbulence of the photo-reaction system during the AOP's development.

In this configuration, the emitted UV radiation, ideally radial, promotes the $\bullet \mathrm{OH}$ radicals generation from the catalyst employed $\left(\mathrm{H}_{2} \mathrm{O}_{2}, \mathrm{O}_{3}\right.$, etc.) inside the liquid to is going to be treated (whit the consequent appearance of the AOP's described above) while it leaves the system. That amount of not retained energy thus increase the time required to degrade the chemical undesired compounds, decreasing the efficiency of the AOP's (because its electric power consume) and therefore, elevating its operating costs.

One way to reduce these losses is to incorporate collectors which have the ability to concentrate again all the emitted radiation into the liquid in treatment, many time as it can be possible. For this purpose, the

*Corresponding author: Tonahtiuh Rendon, Laboratory of Environmental Chemistry, ICUAP, Pubela, Mexico, Tel: +52-12221393806; E-mail: tonahtiuhrendon@hotmail.com

Received November 08, 2016; Accepted November 16, 2016; Published November 22, 2016

Citation: Rendon T, Hernandez F, Castillo J (2016) Comparison of UV Concentration Geometries as an Element Capable for the Increment of the Photocatalytic Efficiency of Advanced Oxidation Processes, Inside the Annular Batch Systems. Int J Waste Resour 6: 257. doi: 10.4172/2252-5211.1000257

Copyright: ( 2016 Rendon T, et al. This is an open-access article distributed under the terms of the Creative Commons Attribution License, which permits unrestricted use, distribution, and reproduction in any medium, provided the original author and source are credited. 
Citation: Rendon T, Hernandez F, Castillo J (2016) Comparison of UV Concentration Geometries as an Element Capable for the Increment of the Photocatalytic Efficiency of Advanced Oxidation Processes, Inside the Annular Batch Systems. Int J Waste Resour 6: 257. doi: $10.4172 / 2252-5211.1000257$

present research examines the application of two potential UV collectors whit a different geometries with the objective to raise the AOP's efficiency.

\section{Cylindrical collector (C.C.)}

The first geometry consists in a cylindrical collector (Figure 2) and is based on the high symmetry that it keeps with the batch system described before (Figure 1). Figure 2 shows that the CC is concentric with the UV source, so all the radiation emitted by the MP-UV lamp, ideally is going to incide normally to the surface of the CC and will be reflected (on the same road) onto the liquid, crossing it at least twice before its interaction with the radiation source (Figure 3).

\section{Compound parabolic concentrator (CPC)}

The second dispositive is formed by the implementation of two compound parabolic collectors opposite onto its aperture area (Figure 4). Since its invention, the CPC have been widely used in AOP's when the operation needs requires the concentration of diffuse UV light. Due to their capacity to concentrate all the radiation that reach its surface onto the circular area of the photo reactor, for which they have been calculated, after one or more reflections [1] these devices do not require of tracking systems and makes more easy the prototypes construction. The pair of CPC is expected to refocus the beams leaving the system at different angle than those found in CC (Figure 5a).

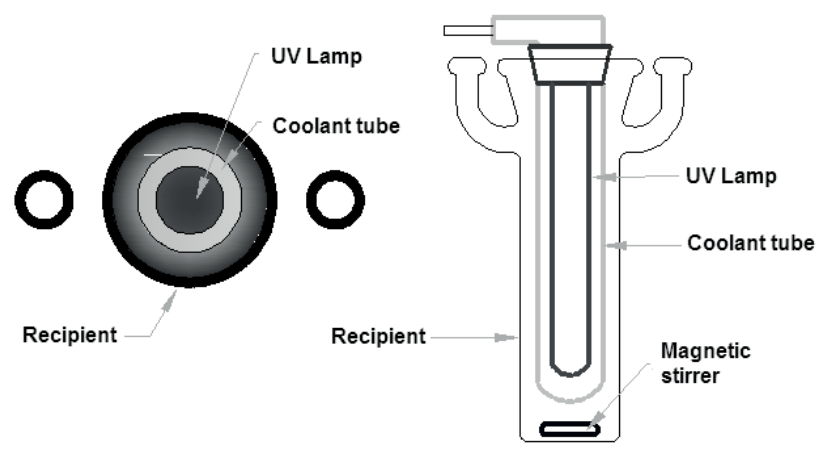

Reactor: Top View

Reactor: Lateral View

Figure 1: Annular batch photo-reactor diagram, for the AOP'S use with UV lamps.

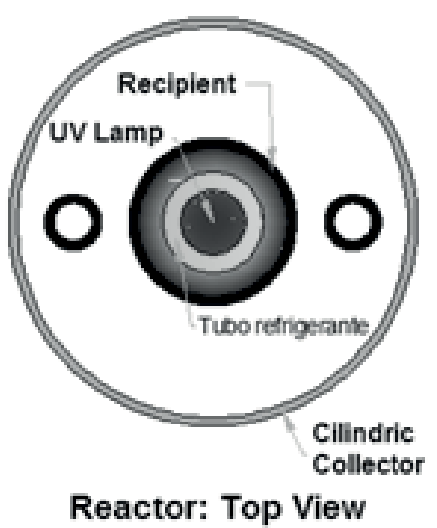

Figure 2: Diagram of annular batch photo-reactor and cylindrical collector implementation.

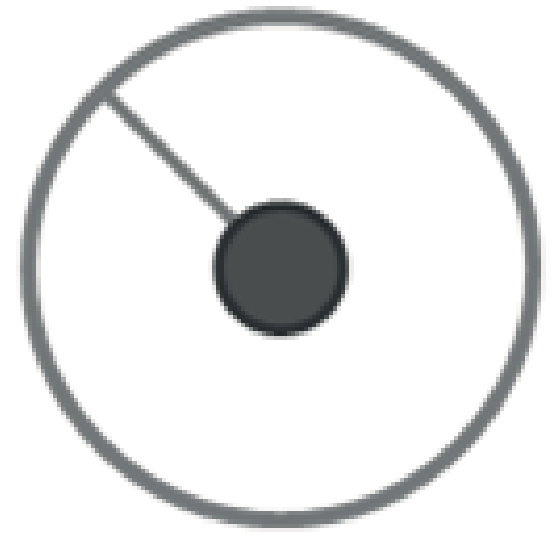

Figure 3: Ray tracing of the CC and the UV source interaction.

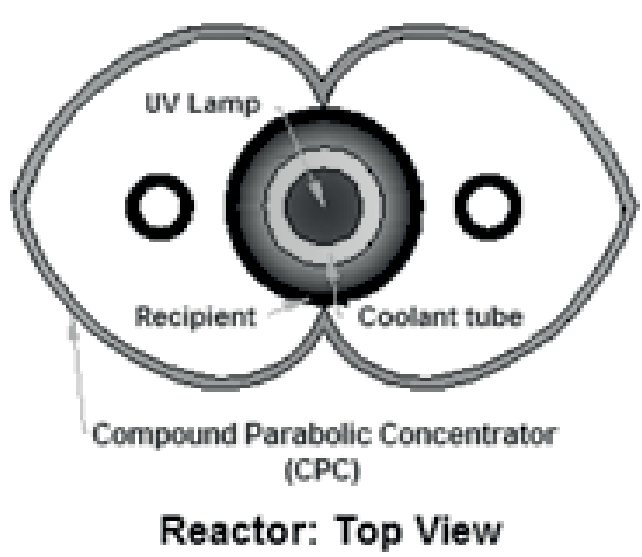

Figure 4: Diagram of annular batch photo-reactor and two opposite complex parabolic concentrators.

The constructed CPC have a concentrator factor equal of one $(\mathrm{CF}=1$, which is defined as the ratio between the calculated circular area and the aperture area of the collector) the maximum angle through which the incoming light can be inside (acceptance angle $\Omega \mathrm{a}$ ) on its surface is of $90^{\circ}$ (Figure $5 \mathrm{~b}$ ).

As is shown in Figure 5, the application of the opposed CPCs, apparently, increase the probability that the beam passes through the liquid to treat a greater number of times (unlike CC) before its interaction whit the mercury lamp.

In based of all mentioned above, the next section describes the methodology employed and the experimental results obtained during the implementation of the mentioned collectors. The analysis was carried out during the degradation of Erionyl Blue A-R acid textile dye in combination whit the use of the homogeneous type AOP's.

\section{Materials and Methods}

For the experimental development two collectors was constructed according with the following geometries:

a) Cylindric collector (CC)

b) Compound parabolic concentrator (CPC) 
Citation: Rendon T, Hernandez F, Castillo J (2016) Comparison of UV Concentration Geometries as an Element Capable for the Increment of the Photocatalytic Efficiency of Advanced Oxidation Processes, Inside the Annular Batch Systems. Int J Waste Resour 6: 257. doi: $10.4172 / 2252-5211.1000257$

Page 3 of 4

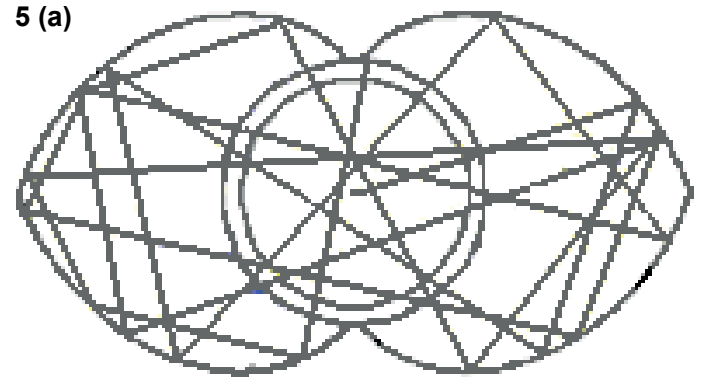

5 (b)

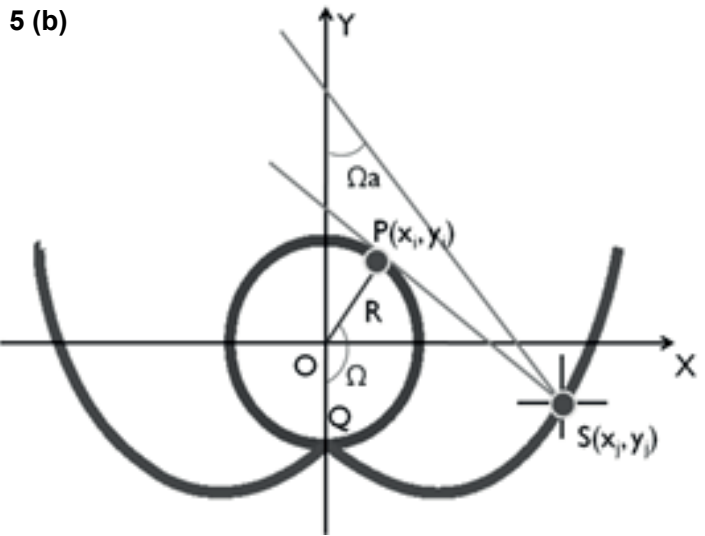

Figure 5: a) Ray trace of one source beam that interact with the twice opposite CPCs. (b) Geometry of the CPC.

Both are made of standard anodized aluminum foil, 16 gauge, and have a total perimeter of $35 \mathrm{~cm}$ of base (which corresponds to the length of the two CPC calculated for the recipient that contains the liquid to be treated, so they have a diameter of $22.28 \mathrm{~cm}$ ) The high of the collectors are of $26.5 \mathrm{~cm}$. Therefore, both devices have a total concentration area of $927.5 \mathrm{~cm}^{2}$.

The cylindrical concentrator was obtained by the use of an industrial foil rolling, and the CPC was approximated by the employ of an industrial bending, making a discrete folds on the aluminum sheet.

For the AOP's experiments, the Acid Blue Erionyl A-R textile dye, at $50 \mathrm{ppm}$, was dissolved in one liter of distilled water. As a source of $\cdot \mathrm{OH}$ radicals, $0.05 \mathrm{~mL}$ of $\mathrm{H}_{2} \mathrm{O}_{2}$ at $50 \%$ was added. The turbulence motion inside the reactor was promoted by the application of a magnetic stirrer. The total treatment operation time was of 5 minutes, extracting the treatment samples each one minute.

The radiation source used is a MP-UV mercury lamp, Heraeus mark, model: PUV-1022. It has $110 \mathrm{~mm}$ of length, $145 \mathrm{~V}, 1000 \mathrm{~W}$ of power and 7.5 A of current consume.

The measurement data was obtained by the use of the equipment listed below:

- $\quad$ Perkin Elmer UV/Vis spectrometer, model: lambda 20.

- $\quad$ Beckman $1 \mathrm{~cm}$ wide quartz cells.

\section{Results and Discussion}

Initially, the AOP's degradation of the Blue erionyl A-R acid colorant was developed on without implementation of any collector. The evolution of the process is showed in Figure 6.
Taking a value of $591 \mathrm{~nm}$ as a wavelength reference [4] the colorant register an initial absorption of 0.54 A.U. At the end of the experimental time, that absorption was of 0.03 A.U. Therefore, the photo-chemical treatment without the use of any kind of collectors has the ability to eliminate the $94.5 \%$ of the compound during the first five minutes of operation. In lengths comprehended between the 280 and $480 \mathrm{~nm}$, is possible to appreciate the generation and the increment of some intermediate compounds that absorbs light at these frequencies.

Then, the same experiment was carried out whit the incorporation of CC following the same methodology and operation conditions mentioned above. Figure 7 describes the annular batch system operation once the device was been implemented.

At the reference wavelength, the process obtains a final value of 0.01 A.U. So the remotion reached whit this configuration, corresponds of a 98.4\%. However, an appreciable generation increment of secondary products inside the interval of $280 \mathrm{~nm}$ and $480 \mathrm{~nm}$ have been observed.

Unlike the previous cases, the double CPC implementation shows a better degradation efficiency, both of the intermediate subproducts generates as those absorbing on the reference wavelength (Figure 8). So, this configuration removes the $100 \%$ of the contaminant after 5 minutes of treatment.

Finally, the Figure 9 shows a comparison of the three configurations studied in this work after the experimental time.

Form the Figure 9, is possible to observe a better experimental performance of the system when a double, and opposite, CPC was used during the AOP's degradation of the Acid Blue Erionyl A-R textile dye, at $50 \mathrm{ppm}$.

\section{Conclusion}

During the present research, two possible collectors, whit different geometries, have been analyzed to promote the efficiency increment of the acid Blue Erionyl A-R acid dye degradation process, during the use of AOP's in homogeneous phase.

The colorant concentration was of $50 \mathrm{ppm}$, dissolved in $1 \mathrm{~L}$ of distilled water, and the performance of the system reported a efficiency raise of 3.4 and $5.5 \%$ (at the reference wavelength of $591 \mathrm{~nm}$ ) when the cylindrical and the two opposite compound parabolic collector was incorporated respectively.

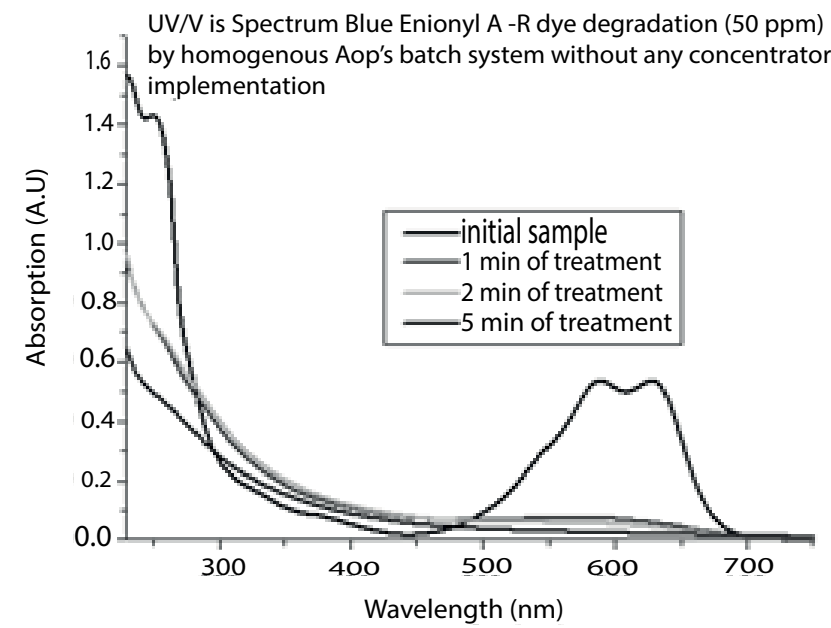

Figure 6: Evolution of the Blue Erionyl A-R acid dye degradation by homogeneous AOP's batch system without any concentrator. 
Citation: Rendon T, Hernandez F, Castillo J (2016) Comparison of UV Concentration Geometries as an Element Capable for the Increment of the Photocatalytic Efficiency of Advanced Oxidation Processes, Inside the Annular Batch Systems. Int J Waste Resour 6: 257. doi: $10.4172 / 2252-5211.1000257$

Page 4 of 4

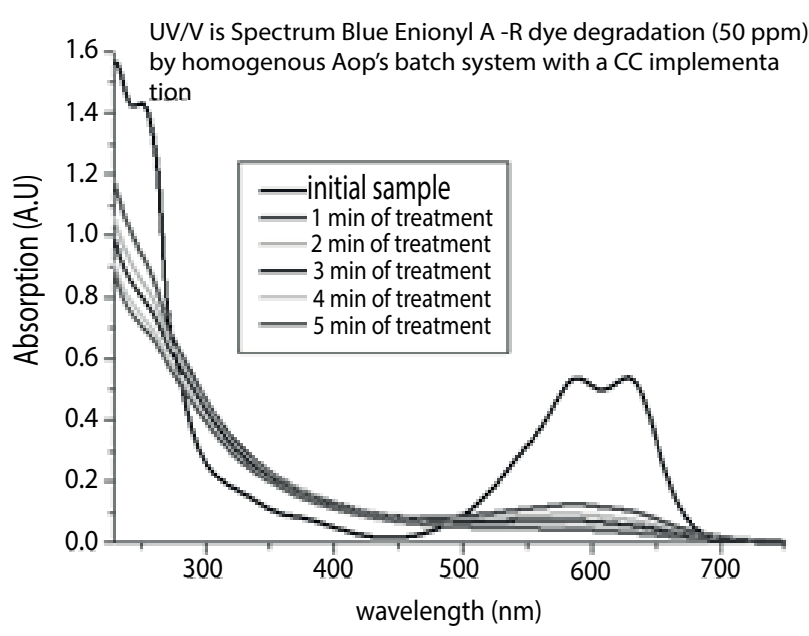

Figure 7: Evolution of the Blue Erionyl A-R acid dye degradation by homogeneous AOP's batch system with CC implementation.

UV/V is Spectrum Blue Enionyl A -R dye degradation (50 ppm)

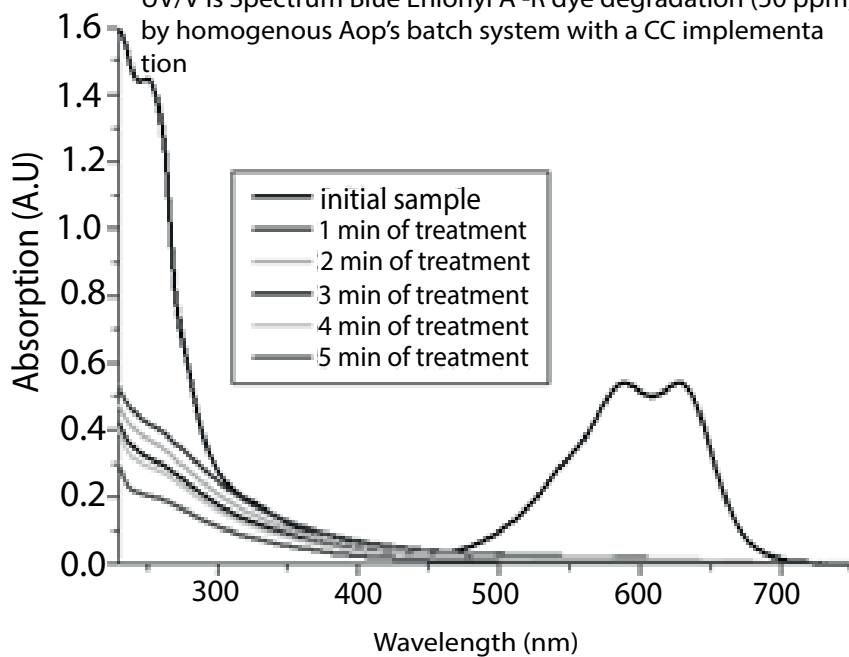

Figure 8: Evolution of the Blue Erionyl A-R acid dye degradation by homogeneous AOP's batch system with CPC implementation.

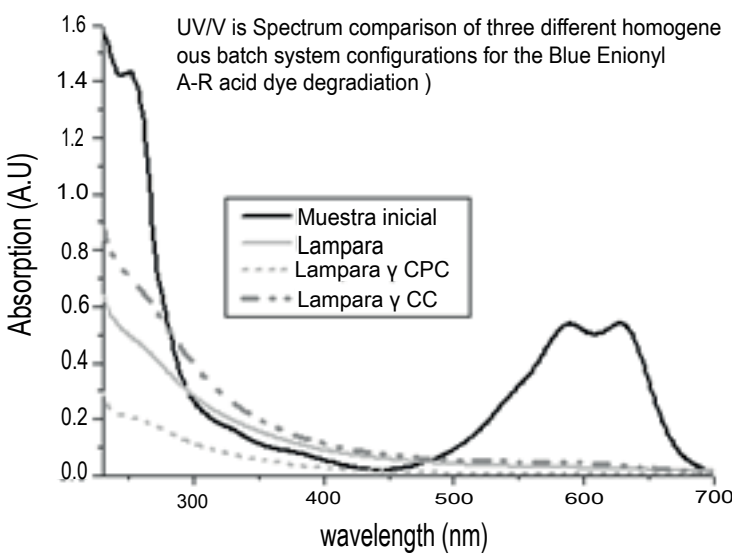

Figure 9: Comparison of the evolution of the homogeneous AOP's batch system with the implementation of CC and CPC collectors, and without them.
Unlike the C.C., experimentally the two opposite CPC demonstrated a greater ability to degrade even the intermediate compounds that are often generated during the 5 minutes of the treatment operation.

The last suggests that there exists an important interaction relation between the UV radiation source, of the reactor, and the incoming reflected rays that cross the liquid medium that is going to be treated. That is traduced into a longer process time, a deficiency of the system and, therefore, in an operation costs increments.

So, a geometry best suited for these kinds of systems would be one that can concentrate all the light that leaves the photochemical recipe, onto the treated aqueous medium, without the interaction between the reflected ray interaction and the UV source diameter. Thus, if possible all the emitted energy will be consumed in the generation of $\bullet \mathrm{OH}$ radicals (useful in the AOP's) and showed little loss due to absorption of the material of which one are made of collectors.

\section{Acknowledgement}

Gratefulness to the CONACYT, ICUAP and the F.C.F.M for the economic support and the facilities for the realization of the present work.

\section{References}

1. Blanco J (2002) Development of solar collectors without concentration for photochemical applications of degradation of persistent contaminants in wáter. Plataforma Solar de Almería, CIEMAT.

2. Jaramillo CA, Taborda G (2006) Photocatalysis: Key aspects for a good removal of contaminants. Rev Univ Caldas enero-diciembre.

3. Garcés LF, Peñuela GA (2007) Waste water treatment of a textile industry with collector solar. Rev Lasallista de Inv 4: 24-31.

4. Hernandez F, Gunter G (2005) Photooxidative treatment of sulfurous water for its potabilization. J Photochem Photobiol 81: 636-640.

5. Farré J, Franch MI, Ayllon JA, Peral J, Domènech X (2005) Biodegradability of treated aqueous solutions of biorecalcitrant pesticides by means of photocatalytic ozonation. Proceedings of the $9^{\text {th }}$ International Conference on Environmental Science and Technology, Rhodes Island, Greece. pp: 402-406.

6. Pera M, García V, Baños M, Giménez J, Esplugues S (2004) Degradation of chlorophenols by means of advanced oxidation processes: a general review. Appl Catal B: Environmental 47: 219-256.

7. Rubalcaba A, Suárez-Ojeda ME, Stüber F, Fortuny A, Bengoa C, et al. (2007) Phenol wastewater remediation: advanced oxidation processes coupled with biological treatment. Water Sci \& Technolog 55: 221-227.

8. Huber M, Canonica S, Park GY, Von GU (2003) Oxidation of pharmaceuticals during ozonation and advanced oxidation processes. Environ Sci Technol 37: 1016-1024.

9. Benitez FJ, Lacero J, Real FJ (2002) Degradation of carbufuran by using ozone, UV radiation and advanced oxidation processes. J Hazardous Materials 89: 51-65

10. www.nwri-usa.org/pdfs/TTChapter-AOPs.pdf 